\section{Revista Brasileira de Administração Científica}

Brazilian Journal of Scientific Administration

Abr a Jun $2021-$ v.12 - n.2
RBADM

ISSN: 2179-684X

\title{
O mercado de criptomoedas no Brasil e os investidores: a caminho de um novo patamar?
}

O mercado das criptomoedas no Brasil, foi impulsionado com o avanço das tecnologias da informação. Porém, este ainda é um mercado considerado inacessível. O artigo teve como objetivo conhecer a intenção e predição de novos investidores à disposição de adesão ao criptomercado, a partir da oferta e acesso à informação sobre o mercado. O processo metodológico do estudo se deu por meio de uma pesquisa quantitativa com aplicação de questionários online a respondentes com pouco ou nenhum conhecimento sobre bitcoins. Os principais resultados do estudo mostram que, há resistência por parte dos respondentes em investir em bitcoins, mas demonstram intenção desde que com acesso as informações disponibilizadas pelo do mercado das criptomoedas.

Palavras-chave: Criptomoedas; Bitcoins; Investimento; Mercado; Informação.

\section{The cryptocurrency market in Brazil and investors: on the way to a new level?}

The cryptocurrency market in Brazil has been boosted by the advance of information technology. However, this is still a market considered inaccessible. The article aimed to know the intention and prediction of new investors willing to join the cryptomarket, from the supply and access to information about the market. The methodological process of the study was carried out through a quantitative research with application of online questionnaires to respondents with little or no knowledge about bitcoins. The main results of the study show that there is resistance on the part of respondents to invest in bitcoins, but they show intention since they have access to information made available by the cryptocurrency market.

Keywords: Cryptomoedas; Bitcoins; Investment; Market; Information.

Topic: Finanças Empresariais

Reviewed anonymously in the process of blind peer
Received: 07/04/2021

Approved: 04/06/2021
Maria Antunizia Gomes (ib)

Instituto Federal do Ceará, Brasil

http://lattes.cnpq.br/0175100794735300

http://orcid.org/0000-0003-1536-3544

antunizia.gomes@yahoo.com.br

Eduardo Paulo Almeida de Sant'Anna (iD)

Universidade Potiguar, Brasil

http://lattes.cnpq.br/6622016595497215

http://orcid.org/0000-0003-3848-1834

epas727@gmail.com
Referencing this:

GOMES, M. A.; SANT'ANNA, E. P. A.. O mercado de criptomoedas no Brasil e os investidores: a caminho de um novo patamar?. Revista Brasileira de Administração Científica, v.12, n.2, p.13-22, 2021. DOI: http://doi.org/10.6008/CBPC2179-684X.2021.002.0002 


\section{INTRODUÇÃO}

Nos últimos anos, criptomoedas surgiram com o desenvolvimento da internet e das novas tecnologias da informação e comunicação. A criação das criptomoedas impôs mudanças nas atividades econômicas entre os usuários da internet, com a expansão destas e o uso da tecnologia de blockchain resultou em um novo patamar econômico na rede mundial de computadores (BARBER et al., 2012; BOHME et al., 2015; GRINBERG, 2012).

Os consumidores estão agora usando cada vez mais criptomoedas como bitcoin ${ }^{1}$ para todos os tipos de pagamentos e estão sendo negociados em mercados de criptomoedas (BARBER et al., 2012; GRINBERG, 2012; KIM et al., 2016; KIM et al., 2017; MARELLA et al., 2017; REID et al., 2013).

Os preços históricos da bitcoin até agora têm mostrado flutuações consideráveis. Elevações em períodos curtos e quedas a taxas alarmantes são frequentes. Especificamente, a moeda bitcoin começou 2017 no valor de USD 997, alcançando o final de dezembro de 2017 a cifra de USD 19.000, potencialmente criando um lucro de quase $1.900 \%$ em um ano. Em junho de 2018, o preço caiu drasticamente para USD 6.000 , o que corresponde a uma perda de aproximadamente $70 \%$ desde dezembro 2017. Esta tendência não pode ser suficientemente explicada pelas teorias macroeconômicas padrão (AALBORG et al., 2019; GERLACH et al., 2018; KRISTOUFEK, 2013; LI et al., 2017).

Criado originalmente, para a função de atuar como um substituto para a moeda fiduciária; entretanto, tem sido usado como reserva de valor, investimento, mercado especulativo e até mercado alternativo onde os mercados tradicionais não alcançam ou não podem, regularmente, alcançar. Ao contrário do mercado monetário real, são atualmente e consideravelmente influenciados pelos investidores de curto prazo, caçadores de tendências, especuladores (KRISTOUFEK, 2013) e muitas atividades de fraude (MOELLER et al., 2017).

Portanto, a análise desses comerciantes, suas atividades e criptomercados torna-se importante para revigorar as criptomoedas e o blockchain (MOELLER et al., 2017). Além disso, a maioria dos investidores em bitcoin determinam sua estratégia de investimento com base nas escassas informações que podem obter porque não há percepção de preços justos neste mercado (AALBORG et al., 2019; KRISTOUFEK, 2013) e há poucas informações sobre os mercados de criptomoedas em comparação com os mercados reais. Diante disso, o presente artigo teve como objetivo entender a intenção de investir no mercado de criptomoedas por meio de acesso à informação sobre o mercado.

Vale ressaltar que, do ponto de vista econômico, uma das principais preocupações sobre o bitcoin é se ele deve ser considerado realmente uma moeda, uma mercadoria ou um estoque. A conclusão de Yermack (2015) é que o bitcoin se comporta como uma ação de alta volatilidade e que a maioria das transações em Bitcoins são destinadas a investimentos especulativos.

\footnotetext{
${ }^{1}$ Bitcoins refere-se a criptomoedas em geral e bitcoin a moeda específica. 


\section{REVISÃO TEÓRICA}

\section{O mercado das criptomoedas}

Recentemente, literatura sobre bitcoin tem prestado muita atenção à dinâmica de preços do Bitcoin e, em particular, à identificação de possíveis direcionadores de preços. Kristoufek (2013; 2015); Kim et al. (2015), Figà-Talamanca et al. (2019) demonstram que o preço do bitcoin e a volatilidade é afetada pelo volume ou número de transações, pelo número de pesquisas no Google sobre o assunto e, buscas na Wikipedia pelo termo bitcoin. Bukovina et al. (2016) registram que o preço do bitcoin está relacionado a uma medida de sentimento obtida através de sites especializados da rede mundial de computadores. Kang et al. (2019) estabeleceram relação direta entre formadores de opinião de fóruns de discussão de bitcoin online e a oscilação de preços das criptomoedas. Para eles tais fóruns se revelam fonte potencialmente rica de informações. Kim et al. (2016), Kim et al. (2017) e Phillips et al. (2018) confirmam tais achados.

\section{O bitcoin e a economia digital}

Uma análise do bitcoin de forma ampla pelo aspecto de evolução tecnológica, percebemos que se trata de sistema que opera num contexto mais amplo das atuais tendências de tecnologia da comunicação e informação. Conectando as pessoas, peer-to-peer (ponto a ponto) cada vez mais e independentemente da origem geográfica ou socioeconômica. O bitcoin permite a conclusão de operações em minutos ao invés de horas ou mesmo dias.

Tabela 1: Principais construtos tecnológicos utilizados em finanças.

\begin{tabular}{|c|c|c|c|}
\hline Tecnologia & Definiçảa & Artigos & Aplicaçöes \\
\hline IOT - Internet of Things & $\begin{array}{l}\text { Internet das Coisas, representa } \\
\text { uma nova era da computaçấo } \\
\text { ubiquas alem da simples interaçâo } \\
\text { conectiva dos objetos. }\end{array}$ & $\begin{array}{l}\text { Pacheco, Klein } \\
\text { e Rhigi (2016) } \\
\text { Lee (2018) }\end{array}$ & $\begin{array}{l}\text { Máquinas de cartáo; } \\
\text { Métodos de pagamento; } \\
\text { Objetos que armazenam } \\
\text { valor. }\end{array}$ \\
\hline $\begin{array}{l}\text { NFC - Near Field } \\
\text { Communication }\end{array}$ & $\begin{array}{l}\text { Forma sem fio de comunicaçâo de } \\
\text { curto alcance, desenvolvida } \\
\text { inicialmente para mobile phones, } \\
\text { baseia-se em RFID. }\end{array}$ & $\begin{array}{l}\text { Juntunem, } \\
\text { Luukkainen e } \\
\text { Tuunainen } \\
(2010)\end{array}$ & $\begin{array}{lr}\text { Cartōes, } & \text { pulseiras, } \\
\text { smartwatches } & \text { e } \\
\text { smartphones } & \text { que } \\
\text { realizam transações por } \\
\text { aprosimação. }\end{array}$ \\
\hline $\begin{array}{l}\text { DLT - Distributed } \\
\text { Ledger Technology } \\
\text { (blockchain) }\end{array}$ & $\begin{array}{l}\text { Banco de dados de registro } \\
\text { diatribuidos que contem um } \\
\text { registro certo e verificável de cada } \\
\text { transação jă feita, as cadeias de } \\
\text { blocos gão protegidas por } \\
\text { criptografia. }\end{array}$ & $\begin{array}{l}\text { Nakamoto } \\
\text { (2008) Swan } \\
(2015) \text { Belmiro } \\
\text { (2018) }\end{array}$ & $\begin{array}{l}\text { Criptomoedas, contratos } \\
\text { inteligentes, expedição de } \\
\text { diplomas e comprovação } \\
\text { de propriedade, } \\
\text { plataforma de Registro de } \\
\text { Titulos. }\end{array}$ \\
\hline Big Data e Analytics & $\begin{array}{l}\text { Análise e interpretação de grande } \\
\text { volume de dados estruturados e } \\
\text { não estruturados. }\end{array}$ & $\begin{array}{l}\text { Lavalle et al. } \\
\text { (2011) } \\
\text { Mohamed e } \\
\text { AlJaroodi } \\
\text { (2014) }\end{array}$ & $\begin{array}{l}\text { Modelos preditivos, } \\
\text { segurança cibernetica e } \\
\text { computaçâo forense em } \\
\text { instituiçoses financeiras. }\end{array}$ \\
\hline Open Banking & $\begin{array}{lcr}\text { Plataformas } & \text { de } & \text { serviços } \\
\text { financeiros } & \text { abertas } & \text { para } \\
\text { desenvolvedores de API. } & & \end{array}$ & $\begin{array}{c}\text { Brodsky e } \\
\text { Oakes (2017) } \\
\text { Guibaud (2015) }\end{array}$ & Integração de plataformas \\
\hline Cloud & $\begin{array}{l}\text { Conjunto de infraestrutura } \\
\text { eacalavel e abstrata capaz de } \\
\text { hospedar aplicaçoos do cliente, } \\
\text { utiliza memória e alta capacidade } \\
\text { de armazenamento de hospedeiros } \\
\text { na rede. }\end{array}$ & $\begin{array}{l}\text { Erdogmus } \\
(2009) \text { Chen } \\
(2011) \text { Gai } \\
(2015)\end{array}$ & Armazenagem de dados \\
\hline $\begin{array}{c}\text { RPA - Robotic Process } \\
\text { Automation }\end{array}$ & $\begin{array}{l}\text { Soluçóes de automaç̧̃o utilizado } \\
\text { robôs que aprendem a executar } \\
\text { tarefas por machine learning. }\end{array}$ & $\begin{array}{l}\text { Wilcocks, } \\
\text { Lacity e Craig } \\
\text { (2017) Van Der } \\
\text { Aalat (2018) }\end{array}$ & $\begin{array}{lr}\text { Aumentar a } & \text { eficiência } \\
\text { operacional } & \mathrm{em} \\
\text { procedimentos } & \mathrm{em} \\
\text { financiamentos, crédito e } \\
\text { hipotecas }\end{array}$ \\
\hline
\end{tabular}

Fonte: Duarte et al. (2020). 
Ao operar de maneira ponto-a-ponto, oferece às pessoas os mesmos movimentos que as empresas globais Uber, Airbnb, Amazon e permitindo que mantenham posições de liderança em seus próprios domínios. Assim, o Bitcoin permite a qualquer pessoa ser seu próprio banco, fornecendo o controle de um movimento popular e empoderando quem não tem banco, globalmente.

As alterações que as novas tecnologias e a transformação digital têm produzido nos mercados e na economia digital, possibilita outra realidade fundamentada em patamar distinto da economia tradicional, oportunizando o novo mercado cripto.

A Tabela 1 acima, oferece a definição das tecnologias impactantes na economia digital atualmente, de Duarte et al. (2020), suas devidas aplicações, com a oferta de peças científicas que embasam cada definição.

\section{O bitcoin e os governos}

Os governos de muitos países estão rejeitando o conceito de criptomoeda ou bitcoins para a economia de seu país, outros, entretanto, se preparam para aceitar este desafio financeiro global (PATEL et al., 2020). Em ambos os casos de aceitar ou rejeitar bitcoins para suas economias, os países têm seu próprio conjunto de razões válidas. Patel et al. (2020), seguem concluindo que bitcoins são moedas segura em termos de armazenamento porque é digital, e devido à alta criptografia durante a execução na rede e transações diretas entre pares.

Mas, por outro lado, como não está vinculado a nenhum sistema centralizado ou autorizado, não é fácil controlar o fluxo de dinheiro. Além das vantagens, o bitcoin também traz algumas armadilhas. Por não haver órgão superior, as transações podem ser afetadas por ameaças e fraudes. A lavagem de dinheiro e o uso de criptomoeda no crime podem afetar diretamente a segurança de qualquer nação, o que será intolerável por qualquer governo.

\section{O sistema bancário}

O setor bancário é uma das primeiras instituições financeiras a ser afetada pelo surgimento da criptomoeda, especialmente da criptomoeda bitcoin mais popular. Os bancos estão desempenhando o papel mais importante no manuseio de dinheiro de qualquer país e em sua credibilidade, conclui relatório Building Global Democracy (2017). Mas por causa do bitcoin, que está negligenciando diretamente a existência de qualquer autoridade entre os pares, também é altamente tecnicamente forte em termos de segurança devido à criptografia, desafiando a forma de funcionamento dos bancos. 'Os bancos também não estão na posição de apenas se sentar e assistir; eles precisam descobrir a maneira de lidar com esse tipo de sistema comparativamente novo de troca de dinheiro'.

\section{As criptomoedas e o mercado de capitais}

O efeito do bitcoin também é inegável sobre os mercados de ações, mesmo que seja de natureza indireta. Ele está entrando em cena em qualquer bolsa de valores com empresas que lidam com Blockchain 
ou outras tecnologias relacionadas à criptomoeda. Também mostrou sua presença nas bolsas de valores mundiais com altos ganhos muitas vezes. A Chicago Mercantile Exchange CME Group, através de sua controlada Bolsa de Chicado, lançou em 2017, contato futuros de bitcoins e em três anos cifras expressivas foram alcançadas (Gráfico 1), embora alguns países, incluindo uma grande economia, a China, tenha banido o Bitcoin por causa de sua natureza altamente volátil.

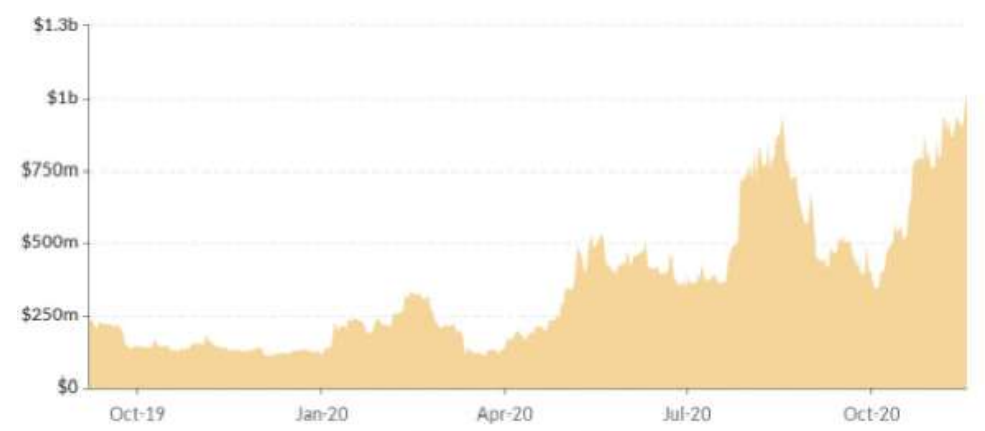

Gráfico 1: Contratos Futuros de Bitcoin em aberto - Bolsa de Chicago/CME Group.

\section{O bitcoin e o funcionamento do blockchain}

A tecnologia blockchain veio do bitcoin, ou seja; a plataforma foi fundada em base tecnológica de criptografia, seu livro-razão distribuído e digital, denominado de blockchain.

No lançamento inicial (janeiro 2009) o bitcoin já foi incorporado com a tecnologia de um blockchain, criado e desenvolvido por Nakamoto (2008). Estava lançada uma nova tecnologia. 'A tecnologia com a maior probabilidade de mudar a próxima década de negócios não é a web social, a big data, as nuvens, a robótica ou mesmo a inteligência artificial; é o blockchain, a tecnologia por trás das moedas digitais como o bitcoin'.

Uma oferta impactante de disrupção no mercado financeiro, a tecnologia blockchain, oferece a oportunidade de um mundo sem cédulas, sem bancos físicos, sem políticas monetárias centralizadas. O valor sendo gerido virtualmente através de um sistema que não tem uma figura de autoridade central e é governado de forma descentralizada e democrática. A matemática faz o ordenamento e controle das operações e do sistema. O sistema inteiro sendo acessado através de computadores e smartfhones.

O Blockchain do bitcoin, uma base de dados distribuída (disponível a qualquer computador no mundo, não controlada por governo ou entidade), criptográfica (todas as transações são verificadas criptograficadamente para assegurar que as pessoas efetivamente podem fazê-la), imutável (a trilha de informação gravada na 'pedra digital' do bitcoin só pode ser adicionada ao longo da cadeia, jamais alterada) e finalmente a prova de trabalho que lida com as transações, agrupa em bloco e encadeia os blocos para formar o blockchain ${ }^{2}$ do bitcoin.

As arquiteturas blockchains e seus ativos nativos estão a caminha de se tornarem a próxima metaaplicação a alavancar a infraestrutura da internet. As moedas globais, inclusive as digitais, computadores mundiais, redes sociais descentralizadas e muitos outros já são servidos pela tecnologia blockchain. O livrorazão distribuído e digital fundado junto com o dinheiro programável, alcançou fama capaz de superar todos

\footnotetext{
${ }^{2}$ Fonte: THE FOUNDATION for Peer to Peer Alternatives. Disponível em: wayback.archive.org/p2pfoundation.ning.com/. Acesso em: 22 fev. 2021. 
os seus objetivos iniciais e ganhar vida própria.

\section{Pesquisas sobre o bitcoin}

Ramona et al. (2019) demonstram que a literatura sobre bitcoins está concentrada em torno de três campos principais: ciências da computação (54,1\%); economia, negócios e finanças (33,5\%); engenharia $(12,7 \%)$.

Em termos de citação, a concentração está no campo econômico, em especial para apresentar os princípios e propriedades de design da plataforma bitcoin para um usuário não técnico e ao público as implicações econômicas para o sistema financeiro Corbet et al. (2018); analisar a volatilidade dos preços do bitcoin Baur et al. (2018), modelar os preços do bitcoin: Guesmi et al. (2019); e metodologias para explorar as capacidades de cobertura do bitcoin Brauneis et al. (2018).

No Brasil, em estudo bibliográfico de artigos publicados em periódicos na CAPES, ao longo de uma década, Araújo (2018) registra o interesse maior em se retratar as características do bitcoin, com estudos focados em "comparativos com índices e propriedades matemáticas" e a busca de se evidenciar o conceito da criptomoeda e seus aspectos principais. Finaliza registrando a escassez de pesquisas sobre a temática nos periódicos nacionais.

Assim, estão formadas as bases da justificativas de nosso estudo, que consistem em analisar esta área carente, ainda não investigada e com objetivo de revelar intenções e predição de atuação e eventual consumo e portabilidade de serviço oferecido por criptomoedas.

\section{METODOLOGIA}

No estudo em questão, investigamos a intenção e predição de portabilidade sobre criptomoedas por parte das pessoas com recursos disponíveis para investimento. Os sujeitos participantes da pesquisa foram jovens e adultos conhecedores o mercado financeiro tradicional (declaram ter ao menos um investimento ativo no mercado financeiro no dia da pesquisa) e declaram não ter conhecimentos ou ter conhecimentos superficiais de criptomoedas.

A fim de alcançar os objetivos propostos, foi realizada uma pesquisa do tipo quantitativa com uso de instrumento de coleta de dados estruturado e divulgação por meio online por meio do google forms. A primeira parte do questionário analisamos seis parâmetros; três de sentido negativo (receio de investir, falta de acesso, dificuldade de acompanhamento do mercado) e três de sentido positivo (ter sucesso com criptomoedas, fazer a primeira transação, crer em modismo para bitcoins).

Na segunda etapa, oferecemos uma questão sobre a intenção de investimento em criptomoedas com três alternativas (sim, não e talvez). A resposta sim levou a oportunidade de registro de intenção de percentuais das disponibilidades para investimento.

No primeiro momento os pesquisados manifestaram opinião sobre assertivas apresentadas na pesquisa. Em seguida, as assertivas foram novamente apresentadas, agora acompanhadas previamente de texto informativo sobre o tema pesquisado. 


\section{RESULTADOS E DISCUSSÃO}

A reação dos pesquisados sobre o receio de investir em bitcoins diminui ao receber um mínimo de informação sobre o mercado específico. A resistência a iniciar operações com criptomoedas diminui à medida que novas informações que o pesquisado recebe sobre o mercado. O sentimento inicial é de que os mercados de bitcoins são inacessíveis fora dos grandes centros financeiros, percepção que é alterada ao receber informações sobre formas e modelos de acesso a este mercado.

Sobre o acompanhamento e evolução do mercado de criptomoedas, o registro inicial é de que é muito difícil (58,9\% concordam). Com informações sobre formas e canais de acompanhamento, esta opinião é alterada $(41,1 \%)$. Entre os pesquisados, $26,8 \%$ acreditavam que poderiam ter sucesso ao acessarem o mercado de criptomoedas; ao receberem um mínimo de informações sobre este mercado a opinião de sucesso tem alteração para 37,5\%.

Quando mudamos o sentido de possibilidades para o sentido concreto de, 'realizar a primeira transação com bitcoins na primeira oportunidade que tiver', obtivemos as respostas como demonstrado adiante:

Tabela 2: Na primeira oportunidade que tiver REALIZAREI uma transação com bitcoin.

\begin{tabular}{l|l|l}
\hline Realizarei & Sem informação de Mercado & Com informação de mercado \\
\hline Concordo & $21,5 \%$ & $34 \%$ \\
\hline Discordo & $51,8 \%$ & $48,2 \%$ \\
\hline
\end{tabular}

A análise dos dados revela evidências da disposição dos pesquisados de, concretamente, realizar sua primeira operação no mercado cripto na primeira oportunidade. Porém, há resistência dos respondentes do estudo a realização de transações com bitcoin, com percentual mais elevado $51,8 \%$ sem informação de mercado.

Registramos ainda que o item 'eu acredito que bitcoin é um modismo, rapidamente vai passar' foi o aspecto dentre os analisados com maior balanceamento nas respostas. Verificamos equilíbrio entre a concordância ou não quanto a assertiva, que se mantém nos dois momentos pesquisados; sem informação e com mínimas informações sobre o mercado de criptomoedas. Quando questionados sobre a disposição real de participar do mercado de criptomoedas, os dados mostram que (Tabela 3).

Tabela 3: Se tiver acesso facilitado, tenho disposição de participar do mercado de bitcoins.

\begin{tabular}{l|l|l}
\hline Disposição em participar & Sem informações do mercado & Com informações do mercado \\
\hline Sim & $33,9 \%$ & $48,2 \%$ \\
\hline Não & $16,1 \%$ & $14,3 \%$ \\
\hline Talvez & $50 \%$ & $37,55 \%$ \\
\hline
\end{tabular}

O acesso facilitado das informações poderá ser um facilitador no processo de investimento, visto que, os respondentes têm intenção de investir no mercado de Bitcoins, principalmente diante de um nível maior de informações do mercado. Dessa forma, importa citar que, para que haja maior intenção e investimento real é importante que as informações sejam disponibilizadas aos investidores, a fim de, inicialmente informar investidores em potencial e posteriormente contribuir para o aumento do interesse em criptomoedas. Patel et al. (2020) corrobora, citando que, as transações por meio do bitcoins são 
transações seguras.

Tabela 4: Percentual das disponibilidades que estaria disposto a investir no mercado cripto.

\begin{tabular}{l|l|l}
\hline$\%$ de investimento & Antes de obter informações & Depois de obter informações \\
\hline 1 a $5 \%$ & $68,3 \%$ & $33,4 \%$ \\
\hline 6 a $10 \%$ & $5,3 \%$ & $22,2 \%$ \\
\hline 11 a $15 \%$ & 15,8 & $37,0 \%$ \\
\hline 16 a $19 \%$ & $5,3 \%$ & $3,7 \%$ \\
\hline Acima $20 \%$ & $5,3 \%$ & $3,7 \%$ \\
\hline
\end{tabular}

Nossos dados revelam aumento dos pesquisados dispostos a participarem do mercado de criptomoedas, após o conhecimento de mínimas informações. Este percentual refere-se principalmente a pesquisados que estavam indecisos (TALVEZ) antes de obterem informações acerca do mercado e alteram posicionamento ao obter informação do mercado.

Quanto aos resultados verificados de 'percentual das disponibilidades financeiras com disposição para investir no mercado de criptomoedas', apurados apenas com respondentes que manifestaram disposição de investir no criptomercado (SIM na questão precedente), constatamos um aumento na intenção.

Os três níveis iniciais atribuídos de respostas, de 1 a 5\%, de 6 a 10\% e de 11 a 15\% de percentual das disponibilidades com disposição de investimento, passam no segundo momento a posição de equilíbrio com $33,4 \%, 22,2 \%$ e $37 \%$ respectivamente, anteriormente o percentual de $68,3 \%$ que o nível 1 (entre 1 e $5 \%$ ) detinha no primeiro momento (respondente sem informações do criptomercado).

O referido estudo revela que o investidor pesquisado demonstra resistência menor à medida em que se informa e aprende sobre o criptomercado. A falta de informação leva a crença de este mercado é inacessível e possível apenas nos grandes centros financeiros, que é difícil acompanhar as criptomoedas e derivativos, mas acreditam que podem ter sucesso em eventual incursão com bitcoins.

Os pesquisados manifestam firme intenção de realizar transação com bitcoins, que ganha ímpeto quando se informam e aprendem sobre este mercado. A falta de informação mantém o sentimento de ceticismo, mas com a possibilidade de "acesso facilitado" a disposição de iniciar no criptomercado é firme. À medida em que as informações são assimiladas a disposição e o percentual de investimento aumentam.

\section{CONCLUSÕES}

O artigo teve como objetivo de revelar a intenção e predição de novos investidores à disposição de adesão ao criptomercado, mostra que a partir da oferta e acesso à informação em ampla escala sobre o funcionamento, variáveis, acompanhamento, métodos, participantes, facilitadores, opções, riscos, dentes outros; é fator que de influência direta sobre a decisão de participação no mercado.

$\mathrm{O}$ acesso à informação demonstrou também influenciar direto e positivamente no aumento da intenção de investimento. Ressaltando a importância da disseminação de informações contínuas sobre o mercado de criptomoedas no contexto brasileiro.

Informação e conhecimento, se revelam pontos importantes para o crescimento do criptomercado e a regra Burniske-Tatar sempre deve permear seus negócios; "não invista em bitcoin, ether, ou qualquer 
outro criptoativo apenas porque ele dobrou ou triplicou na última semana. Antes de investir, seja capaz de explicar o básico sobre um ativo para um amigo e certificar-se de ele se encaixa bem, considerados o perfil, risco e os objetivos do seu portfólio de investimento" (BURNISKE et al., 2019).

Reconhecemos as limitações de nossos estudos e registramos que novos e mais abrangentes ensaios devem ser realizados com a utilização de constructos que permitam análises e estatísticas correlatas.

\section{REFERÊNCIAS}

AALBORG, H. A.; MOLNA'R, P.; VRIES, J. E.. What can explain the price, volatility and trading volume of Bitcoin? Finance Research Letters, v.29, p.255-265, 2019. DOI: http://doi.org/10.1016/j.frl.2018.08.010

ARAÚJO, V. H. R.. Bitcoin: uma análise das pesquisas de contabilidade e finanças publicadas em periódicos. Monografia (Bacharelado em Ciências Contábeis) Universidade Federal de Uberlândia, Ituiutaba, 2018.

BARBER, S.; BOYEN, X.; SHI, E.; UZUN, E.. Bitter to Better: how to make bitcoin a Better Currency. In: KEROMYTIS, A. D.. Financial Cryptography and Data Security. Lecture Notes in Computer Science. Berlin: Springer, 2012. DOI: https://doi.org/10.1007/978-3-642-32946-3 29

BAUR, D. G.; DIMPFL, T.; KUCK, K.. Bitcoin, gold and the US dólar: A replication and extension. Finance Research Letters, n.25, p.103-110, 2018. DOI: https://doi.org/10.1016/j.frl.2017.10.012

BOHME, R.; CHRISTIN, N.; EDELMAN, B.; MOORE, T.. Bitcoin: Economics, technology, and governance. Journal of Economic Perspectives, v.29, p.213-238, 2015.

BRAUNEIS, A.; MESTEL, R.. Price discovery of cryptocurrencies: Bitcoin and beyond. Economics Letters, v.165, p.58-61, 2018.

BUKOVINA, J.; MARTI ${ }^{\vee}$ CEK, M.. Sentiment and bitcoin volatility. Technical report, Mendel University in Brno, Faculty of Business and Economics, 2016.

BURNISKE, C.; TATAR, J.. Criptoativos: o guia do investidor inovador para o bitcoin e além. Rio de Janeiro: Alta Books, 2019.

CORBET, S.; LUCEY, B.; YAROVAYA, L.. Datestamping the Bitcoin and Ethereum bubbles. Finance Research Letters, v.26, p.81-88, 2018.

DUARTE, R. G.; LUFT, M. C. M. S.; FREITAS, F. C. H. P.; SILVA, M. R. S.; MATOS JÚNIOR, J. E.. Transformação digital em finanças: cenário de duas perspectivas teóricas. In: CONGRESSO TRANSFORMAÇÃO DIGITAL 2020. Anais. Fundação Getúlio Vargas, 2020.

FIGÀ-TALAMANCA, G.; PATACCA, M.. Does market attention affect Bitcoin returns and volatility?. Decisions Econ. Finan., v.42, p.135-155, 2019.

GERLACH, J. C.; DEMOS, G.; SORNETTE, D.. Dissection of Bitcoin's Multiscale Bubble History from January 2012 to February 2018. Royal Society Open Science Journal, v.6, n.7, 2019. DOI: http://dx.doi.org/10.1098/rsos.180643
GRINBERG, R.. Bitcoin: An innovative alternative digital currency. Hastings Science and Technology Law Journal, v.4, n.1, p.159-207, 2012.

GUESMI, K.; SAADI, S.; ABID, I.; FTITI, Z.. Portfolio diversification with virtual currency: Evidence from bitcoin. International Review of Financial Analysis, v.63, p.431-437, 2019.

KANG, K.; CHOO, J.; KIM WHOSE, Y.. Opinion Matters? Analyzing Relationships Between Bitcoin Prices and User Groups in Online Community. Social Science Computer Review, v.38, n.6, p.1-17, 2019. DOI: https://doi.org/10.1177/0894439319840716

KIM, M.; KANG, K.; PARK, D.; CHOO, J.; ELMQVIST, N.. Topiclens: Efficient multi-level visual topic exploration of large-scale document collections. IEEE Transactions on Visualization and Computer Graphics, v.23, p.151-160, 2017.

KIM, Y. B.; KIM, J. G.; KIM, W.; IM, J. H.; KIM, T. H.; KANG, S. J.. Predicting fluctuations in cryptocurrency transactions based on user comments and replies. PLoS ONE, v.11, n.8, p.e0161197, 2016. DOI: https://doi.org/10.1371/journal.pone.0161197

KIM, Y. B.; LEE, S. H.; KANG, S. J.; CHOI, M. J.; LEE, J.; KIM, C. $\mathrm{H}$.. Virtual world currency value fluctuation prediction system based on user sentiment analysis. PLoS ONE, v.10, p.e0132944, 2015. DOI: https://doi.org/10.1371/journal.pone.0132944

KRISTOUFEK, L.. Bitcoin meets Google Trends and Wikipedia: Quantifying the relationship between phenomena of the Internet era. Scientific Reports, v.3, p.3415, 2013.

KRISTOUFEK, L.. What are the main drivers of the bitcoin price? Evidence from wavelet coherence analysis. PLoS ONE 10, v.4, p.e0123923, 2015.

LI, X.; WANG, C. A.. The technology and economic determinants of cryptocurrency exchange rates: The case of Bitcoin. Decision Support Systems, v.95, p.49-60, 2017.

MARELLA, V.; LINDMAN, J.; ROSSI, M.; TUUNAINEN, V.. Bitcoin: a social movement under attack. Selected Papers of the IRIS, n.8, v.1, 2017.

MOELLER, K.; MUNKSGAARD, R.; DEMANT, J.. Flow my FE the vendor said: Exploring violent and fraudulent resource exchanges on cryptomarkets for illicit drugs. American Behavioral Scientist, v.61, p.1427-1450, 2017. 
NAKAMOTO, S.. Bitcoin: A peer-to-peer electronic cash system. Retrived from Bitcoin Project Website, 2008.

PATEL, H.; BURLA, S.; MANJULA, J. B.. Impact of Bitcoin on the World Economy: Opportunities and Challenges. In: RAIPUT, D. S.. Transforming businesses with bitcoin mining and blockchain applications, c9. Nova Delhi: IGI Global, 2020. DOI: http://doi.org/10.4018/978-1-7998-0186

PHILLIPS, R. C.; GORSE, D.. Predicting cryptocurrency price bubbles using social media data and epidemic modelling. Computational Intelligence. IEEE Symposium Series on
Piscataway. Institute of Electrical and Electronics Engineers, 2017. DOI: http://doi.org/10.1109/SSCl.2017.8280809

RAMONA, O.; CRISTINA, M. S.; RALUCA, S.. Bitcoin in the scientific literature: A bibliometric study. Studies in Business and Economics, v.14, p.3, 2019.

REID, F.; HARRIGAN, M.. An analysis of anonymity in the Bitcoin system. In: ALTSHULER, Y. Y.. Security and Privacy in Social Networks. New York: Springer, 2013. p.197-223. DOI: http://doi.org/10.1007/978-1-4614-4139-7 10

YERMACK, D.. Is bitcoin a real currency? An economic appraisal. Amsterdam: Elsevier, 2015.

A CBPC - Companhia Brasileira de Produção Científica (CNPJ: 11.221.422/0001-03) detém os direitos materiais desta publicação. Os direitos referem-se à publicação do trabalho em qualquer parte do mundo, incluindo os direitos às renovações, expansões e disseminações da contribuição, bem como outros direitos subsidiários. Todos os trabalhos publicados eletronicamente poderão posteriormente ser publicados em coletâneas impressas sob coordenação da Sustenere Publishing, da Companhia Brasileira de Produção Científica e seus parceiros autorizados. Os (as) autores (as) preservam os direitos autorais, mas não têm permissão para a publicação da contribuição em outro meio, impresso ou digital, em português ou em tradução. 